\title{
EXISTENCE AND UNIQUENESS OF A CLASSICAL SOLUTION TO A FUNCTIONAL-DIFFERENTIAL ABSTRACT NONLOCAL CAUCHY PROBLEM
}

\author{
LUDWIK BYSZEWSKI \\ Cracow University of Technology \\ Institute of Mathematics \\ Warszawska 24 \\ 31-155 Cracow, Poland
}

(Received November, 1997; Revised April, 1998)

The aim of this paper is to investigate the existence and uniqueness of a classical solution to a functional-differential abstract nonlocal Cauchy problem in a general Banach space. For this purpose, a special kind of a mild solution is introduced and the Banach contraction theorem and a modified Picard method are applied.

Key words: Abstract Cauchy Problem, Ordinary Functional-Differential Equation, Nonlocal Condition, Existence and Uniqueness of a Classical Solution, Mild Solution, Banach Contraction Theorem, Picard Method.

AMS subject classifications: $34 \mathrm{G} 20,34 \mathrm{~K} 30,34 \mathrm{~A} 12,34 \mathrm{~A} 34,47 \mathrm{H} 10$, 34A45, 34G99.

\section{Introduction}

We present four theorems (Theorems 2.1-2.4) on the existence and uniqueness of a classical solution to a functional-differential abstract nonlocal Cauchy problem in an arbitrary Banach space and give an approximation of the solution to the nonlocal problem. In the proofs of the theorems, we introduce a special kind of a mild solution and apply the Banach contraction theorem and a modified Picard method of successive approximations.

The functional-differential nonlocal problem, studied in this paper is of the form:

$$
\begin{gathered}
u^{\prime}(t)=f(t, u(t), u(a(t))), \quad t \in I, \\
u\left(t_{0}\right)+\sum_{k=1}^{p} c_{k} u\left(t_{k}\right)=x_{0},
\end{gathered}
$$

where $I:=\left[t_{0}, t_{0}+T\right], t_{0}<t_{1}<\ldots<t_{p} \leq t_{0}+T, T>0 ; f: I \times E^{2} \rightarrow E$ and $a: I \rightarrow I$ are given functions satisfying some assumptions; $E$ is a Banach space with norm $\|\cdot\|, x_{0} \in E, c_{k} \neq 0 \quad(k=1, \ldots, p)$ and $p \in \mathbb{N}$.

Printed in the U.S.A. (C)1999 by North Atlantic Science Publishing Company 
The results obtained are generalizations and continuations of those, reported previously in [1-4], with the nonlocal condition of type (1.2). Moreover, the results of the paper include, among other things, a special kind of a mild solution to nonlocal problem (1.1)-(1.2). Therefore, throughout the proofs of the theorems, we apply properties of function $f$ in a greater measure than in [1-3]. Consequently, in contrast with [1-3], now, even if $T$ is an arbitrary positive constant, then $c_{k}(k=1, \ldots, p)$ from the nonlocal condition (1.2) can satisfy the inequalities $\left|c_{k}\right|>1(k=1, \ldots, p)$. The special kind of a mild solution in this paper is a modification of a mild solution introduced by the author (in [5]), for nonlocal evolution problems. In the case when $c_{k}=$ $0(k=1, \ldots, p)$ and the right-hand side of the functional-differential equation does not depend on the functional argument, some results of Theorem 2.4 are reduced to those (given in [6]) on the existence and uniqueness of a classical solution to the abstract Cauchy problem with the standard initial condition.

If $c_{k} \neq 0(k=1, \ldots, p)$ then the results of the paper can be applied in kinematics to determine the evolution $t \rightarrow u(t)$ of the location of a physical object for which we do not know the positions $u\left(t_{0}\right), u\left(t_{1}\right), \ldots, u\left(t_{p}\right)$, but we know that the nonlocal condition (1.2) holds. Consequently, to describe some physical phenomena, the nonlocal condition can be more useful than the standard initial condition $u\left(t_{0}\right)=x_{0}$.

\section{Theorems About the Existence and Uniqueness of a Classical Solution}

By $X$, we denote the Banach space $C(I, E)$ with the standard norm $\|\cdot\|_{X} \cdot$ So,

$$
\|w\|_{X}:=\sup _{t \in I}\|w(t)\|, w \in X .
$$

Assume that $\sum_{k=1}^{p} c_{k} \neq-1$. A function $u \in X$, satisfying the integral equation

$$
\begin{gathered}
u(t)=\left(x_{0}-\sum_{k=1}^{p} c_{k} \int_{t_{0}}^{t_{k}} f(\tau, u(\tau), u(a(\tau))) d \tau\right) /\left(1+\sum_{k=1}^{p} c_{k}\right) \\
+\int_{t_{0}}^{t} f(\tau, u(\tau), u(a(\tau)) d \tau, \quad t \in I,
\end{gathered}
$$

is said to be a mild solution of the nonlocal problem (1.1)-(1.2).

A function $u: I \rightarrow E$ is said to be a classical solution of the nonlocal problem (1.1)(1.2) if

(i) $\quad u$ is continuous on $I$ and continuously differentiable on $I$,

(ii) $\quad u^{\prime}(t)=f(t, u(t), u(a(t)))$ for $t \in I$

and

(iii) $u\left(t_{0}\right)+\sum_{k=1}^{p} c_{k} u\left(t_{k}\right)=x_{0}$.

Theorem 2.1: Suppose that $f: I \times E^{2} \rightarrow E, a: I \rightarrow I$ and $\sum_{k=1}^{p} c_{k} \neq-1$. If $u$ is a classical solution of the nonlocal problem (1.1)-(1.2), then $u$ is a mild solution of this problem. 
Proof: Let $u$ be a classical solution of the nonlocal problem (1.1)-(1.2). Then $u$ satisfies equation (1.1) and, consequently,

From (2.2),

$$
u(t)=u\left(t_{0}\right)+\int_{t_{0}}^{t} f(\tau, u(\tau), u(a(\tau))) d \tau, \quad t \in I
$$

$$
u\left(t_{k}\right)=u\left(t_{0}\right)+\int_{t_{0}}^{t_{k}} f(\tau, u(\tau), u(a(\tau))) d \tau \quad(k=1, \ldots, p) .
$$

By (1.2) and (2.3),

$$
u\left(t_{0}\right)+\sum_{k=1}^{p} c_{k}\left[u\left(t_{0}\right)+\int_{t_{0}}^{t_{k}} f(\tau, u(\tau), u(a(\tau))) d \tau\right]=x_{0} .
$$

Since $\sum_{k=1}^{p} c_{k} \neq-1$, then $(2.4)$ implies

$$
u\left(t_{0}\right)=\left(x_{0}-\sum_{k=1}^{p} c_{k} \int_{t_{0}}^{t_{k}} f(\tau, u(\tau), u(a(\tau))) d \tau\right) /\left(1+\sum_{k=1}^{p} c_{k}\right) .
$$

From (2.2) and (2.5), we obtain that $u$ is a mild solution of the nonlocal problem (1.1)-(1.2). The proof of Theorem 2.1 is complete.

Theorem 2.2: Suppose that $f \in C\left(I \times E^{2}, E\right), a: I \rightarrow I$ and $\sum_{k=1}^{p} c_{k} \neq-1$. If $u$ is a mild solution of the nonlocal problem (1.1)-(1.2) then $u$ is a classical solution of this problem.

Proof: Let $u$ be a mild solution of the nonlocal problem (1.1)-(1.2). Then $u$ satisfies equation (1.1) and, from the continuity of $f, u \in C^{1}(I, E)$. Now, we will show that $u$ satisfies the nonlocal condition (1.2). For this purpose, observe that, by $(2.1)$

and

$$
u\left(t_{0}\right)=\left(x_{0}-\sum_{k=1}^{p} c_{k} \int_{t_{0}}^{t_{k}} f(\tau, u(\tau), u(a(\tau))) d \tau\right) /\left(1+\sum_{k=1}^{p} c_{k}\right)
$$

$$
\begin{gathered}
u\left(t_{i}\right)=\left(x_{0}-\sum_{k=1}^{p} c_{k} \int_{t_{0}}^{t_{k}} f(\tau, u(\tau), u(a(\tau))) d \tau\right) /\left(1+\sum_{k=1}^{p} c_{k}\right) \\
+\int_{t_{0}}^{t_{i}} f(\tau, u(\tau), u(a(\tau))) d \tau \quad(i=1, \ldots, p) .
\end{gathered}
$$

From (2.6) and (2.7), and from some computations, 


$$
\begin{gathered}
u\left(t_{0}\right)+\sum_{i=1}^{p} c_{i} u\left(t_{i}\right)=\left(x_{0}-\sum_{k=1}^{p} c_{k} \int_{t_{0}}^{t_{k}} f(\tau, u(\tau), u(a(\tau))) d \tau\right) \\
+\sum_{i=1}^{p} c_{i} \int_{t_{0}}^{t_{i}} f(\tau, u(\tau), u(a(\tau))) d \tau=x_{0} .
\end{gathered}
$$

Therefore, the proof of Theorem 2.2 is complete.

As a consequence of Theorems 2.1 and 2.2, we obtain:

Theorem 2.3: Suppose that $f \in C\left(I \times E^{2}, E\right), a: I \rightarrow I$ and $\sum_{k=1}^{p} c_{k} \neq-1$. Then $u$ is the unique classical solution of the nonlocal problem (1.1)-(1.2) if and only if $u$ is the unique mild solution of this problem.

Now, we will prove the main theorem of the paper.

Theorem 2.4: Assume that:

(i) $\quad a \in C(I, I), f: I \times E^{2} \rightarrow E$ is continuous with respect to the first variable on $I$ and there is $L>0$ such that

$$
\begin{gathered}
\left\|f\left(s, z_{1}, z_{2}\right)-f\left(s, \tilde{z}_{1}, \tilde{z}_{2}\right)\right\| \leq L \sum_{i=1}^{2}\left\|z_{i}-\tilde{z}_{i}\right\| \\
\text { for } s \in I, z_{i}, \tilde{z}_{i} \in E \quad(i=1,2),
\end{gathered}
$$

$\underset{\text { and }}{\text { (ii) }} \sum_{k=1}^{p} c_{k} \neq-1$

(iii)

$$
2 \operatorname{LT}\left(1+\left|\left(\sum_{k=1}^{p} c_{k}\right) /\left(1+\sum_{k=1}^{p} c_{k}\right)\right|\right)<1
$$

Then the nonlocal Cauchy problem (1.1)-(1.2) has a unique classical solution $u$. Moreover, the successive approximations $u_{n}(n=0,1,2, \ldots)$, defined by the formulas

and

$$
u_{0}(t):=x_{0} \text { for } t \in I
$$

$$
\begin{aligned}
u_{n+1}(t): & =\left(x_{0}-\sum_{k=1}^{p} c_{k} \int_{t_{0}}^{t_{k}} f\left(\tau, u_{n}(\tau), u_{n}(a(\tau))\right) d \tau\right) /\left(1+\sum_{k=1}^{p} c_{k}\right) \\
& +\int_{t_{0}}^{t} f\left(\tau, u_{n}(\tau), u_{n}(a(\tau))\right) \text { for } t \in I \quad(n=0,1,2, \ldots),
\end{aligned}
$$

converge uniformly on I to the unique classical solution $u$.

Proof: Introduce an operator $A$ by the formula

$$
(A w)(t):=\left(x_{0}-\sum_{k=1}^{p} c_{k} \int_{t_{0}}^{t_{k}} f(\tau, w(\tau), w(a(\tau))) d \tau\right) /\left(1+\sum_{k=1}^{p} c_{k}\right)
$$




$$
+\int_{t_{0}}^{t} f(\tau, w(\tau), w(a(\tau))) d \tau, w \in X, t \in I
$$

It is easy to see that

$$
A: X \rightarrow X \text {. }
$$

Now, we will show that $A$ is a contraction on $X$. For this purpose observe that

$$
\begin{gathered}
(A w)(t)-(A \widetilde{w})(t) \\
=\left(-\sum_{k=1}^{p} c_{k} \int_{t_{0}}^{t_{k}}[f(\tau, w(\tau), w(a(\tau)))-f(\tau, \widetilde{w}(\tau), \widetilde{w}(a(\tau)))] d \tau\right) /\left(1+\sum_{k=1}^{p} c_{k}\right) \\
\quad+\int_{t_{0}}^{t}[f(\tau, w(\tau), w(a(\tau)))-f(\tau, \tilde{w}(\tau), \widetilde{w}(a(\tau)))] d \tau, w, \widetilde{w} \in X, \quad t \in I .
\end{gathered}
$$

From (2.13) and (2.8),

$$
\|(A w)(t)-(A \widetilde{w})(t)\|
$$

$$
\leq 2 \operatorname{LT}\left(1+\left|\left(\sum_{k=1}^{p} c_{k}\right) /\left(1+\sum_{k=1}^{p} c_{k}\right)\right|\right)\|w-\tilde{w}\|_{X}, \quad w, \widetilde{w} \in X, \quad t \in I .
$$

Let

$$
q:=2 L T\left(1+\left|\left(\sum_{k=1}^{p} c_{k}\right) /\left(1+\sum_{k=1}^{p} c_{k}\right)\right|\right)
$$

Then, by (2.14), (2.15) and assumption (iii),

$$
\|A w-A \widetilde{w}\|_{X} \leq q\|w-\widetilde{w}\|_{X} \text { for } w, \widetilde{w} \in X
$$

with $0<q<1$.

Consequently, by (2.12) and (2.16), operator $A$ satisfies all the assumptions of the Banach contraction theorem. Therefore, in space $X$ there is only one fixed point $u$ of $A$ and this point is the mild solution of the nonlocal problem (1.1)-(1.2). Consequently, from Theorem 2.3,u is the unique classical solution of the nonlocal problem (1.1)(1.2).

Now, we will prove the second part of the thesis of Theorem 2.4. To this end, observe that by $(2.10)$ and $(2.9)$,

$$
\begin{gathered}
\left\|u_{1}-u_{0}\right\|_{X}=\sup _{t \in I}\left\|u_{1}(t)-u_{0}(t)\right\| \\
\leq\left\|\left(-\sum_{k=1}^{p} c_{k} \int_{t_{0}}^{t_{k}} f\left(\tau, u_{0}(\tau), u_{0}(a(\tau))\right) d \tau\right) /\left(1+\sum_{k=1}^{p} c_{k}\right)\right\|
\end{gathered}
$$


where

$$
\begin{aligned}
& +\sup _{t \in I} \| \int_{t_{0}}^{t} f\left(\tau, u_{0}(\tau), u_{0}(a(\tau))\right) d \tau \mid \\
& \leq M T\left(1+\left|\left(\sum_{k=1}^{p} c_{k}\right) /\left(1+\sum_{k=1}^{p} c_{k}\right)\right|\right)
\end{aligned}
$$

$$
M:=\sup \{\|f(\tau, w(\tau), w(a(\tau)))\|: w \in X, \tau \in I\}
$$

Next, assume that

$$
\begin{gathered}
\left\|u_{n}-u_{n-1}\right\|_{X} \leq M T\left(1+\left|\left(\sum_{k=1}^{p} c_{k}\right) /\left(1+\sum_{k=1}^{p} c_{k}\right)\right|\right) \\
\cdot\left[2 L T\left(1+\left|\left(\sum_{k=1}^{p} c_{k}\right) /\left(1+\sum_{k=1}^{p} c_{k}\right)\right|\right)\right]^{n-1}
\end{gathered}
$$

for some natural $n \geq 2$.

Then, by (2.10), (2.9), (2.8) and (2.18),

$$
\left\|u_{n+1}-u_{n}\right\|_{X}=\sup _{t \in I}\left\|u_{n+1}(t)-u_{n}(t)\right\|
$$

$$
\begin{gathered}
\leq\left\|\left(-\sum_{k=1}^{p} c_{k} \int_{t_{0}}^{t_{k}}\left[f\left(\tau, u_{n}(\tau), u_{n}(a(\tau))\right)-f\left(\tau, u_{n-1}(\tau), u_{n-1}(a(\tau))\right)\right] d \tau\right) /\left(1+\sum_{k=1}^{p} c_{k}\right)\right\| \\
+\sup _{t \in I}\left\|\int_{t_{0}}^{t}\left[f\left(\tau, u_{n}(\tau), u_{n}(a(\tau))\right)-f\left(\tau, u_{n-1}(\tau), u_{n-1}(a(\tau))\right)\right] d \tau\right\| \\
\leq 2 L T\left(1+\left|\left(\sum_{k=1}^{p} c_{k}\right) /\left(1+\sum_{k=1}^{p} c_{k}\right)\right|\right)\left\|u_{n}-u_{n-1}\right\| X \\
\leq \operatorname{MT}\left(1+\left|\left(\sum_{k=1}^{p} c_{k}\right) /\left(1+\sum_{k=1}^{p} c_{k}\right)\right|\right) \cdot\left[2 L T\left(1+\left|\left(\sum_{k=1}^{p} c_{k}\right) /\left(1+\sum_{k=1}^{p} c_{k}\right)\right|\right)^{n} .\right.
\end{gathered}
$$

Therefore, from (2.17), (2.18), (2.19), and from mathematical induction,

$$
\begin{gathered}
\left\|u_{n}-u_{n-1}\right\|_{X} \leq M T\left(1+\left|\left(\sum_{k=1}^{p} c_{k}\right) /\left(1+\sum_{k=1}^{p} c_{k}\right)\right|\right) \\
\cdot\left[2 L T\left(1+\left|\left(\sum_{k=1}^{p} c_{k}\right) /\left(1+\sum_{k=1}^{p} c_{k}\right)\right|\right)\right]^{n-1}
\end{gathered}
$$


for all $n=1,2, \ldots$.

Inequalities (2.20) and assumption (iii) imply, by the Weierstrass theorem, the uniform convergence of the series

$$
u_{1}+\sum_{n=1}^{\infty}\left(u_{n+1}-u_{n}\right)
$$

on the interval $I$ and, consequently, the uniform convergence of the sequence $u_{n}$ on $I$. Let

$$
u_{*}(t):=\lim _{n \rightarrow \infty} u_{n}(t) \text { for } t \in I .
$$

Since $u_{n}$ tends uniformly to $u_{*}$ on $I$ then, by (2.9), (2.10) and (2.8), $u_{*}$ is a classical solution of the nonlocal problem (1.1)-(1.2) on $I$. But, from the first part of the thesis of Theorem 2.4, we know that there exists only one classical solution $u$ of the nonlocal problem (1.1)-(1.2) on $I$. So, $u_{*}=u$ on $I$.

The proof of Theorem 2.4 is complete.

\section{References}

[1] Byszewski, L. and Lakshmikantham, V., Theorem about the existence and uniqueness of a solution of a nonlocal abstract Cauchy problem in a Banach space, Appl. Anal. 40 (1990), 11-19.

[2] Byszewski, L., Existence of approximate solution to abstract nonlocal Cauchy problem, J. of Appl. Math. and Stoch. Anal. 5.4 (1992), 363-374.

[3] Byszewski, L., Differential and Functional-Differential Problems with Nonlocal Conditions, Cracow University of Technology, Monograph 184, Cracow 1995.

[4] Byszewski, L., On weak solutions of functional-differential abstract nonlocal Cauchy problem, Annales Polonici Math. 65 (1997), 163-170.

[5] Byszewski, L., Application of properties of the right-hand sides of evolution equations to an investigation of nonlocal evolution problems, Nonl. Anal.: Theory, Methods and Appl. (1998), in press.

[6] Lakshmikantham, V. and Leela, S., Nonlinear Differential Equations in Abstract Spaces, Pergamon Press, Oxford, New York, Toronto, Sydney, Paris, Frankfurt 1981. 


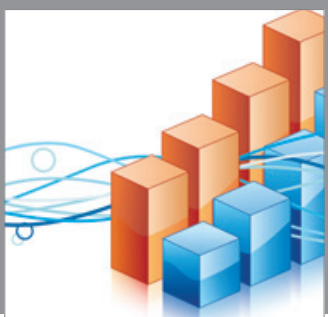

Advances in

Operations Research

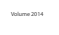

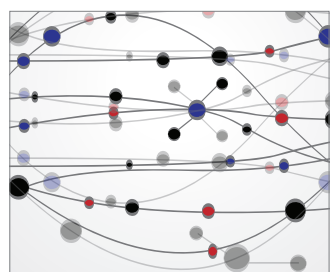

\section{The Scientific} World Journal
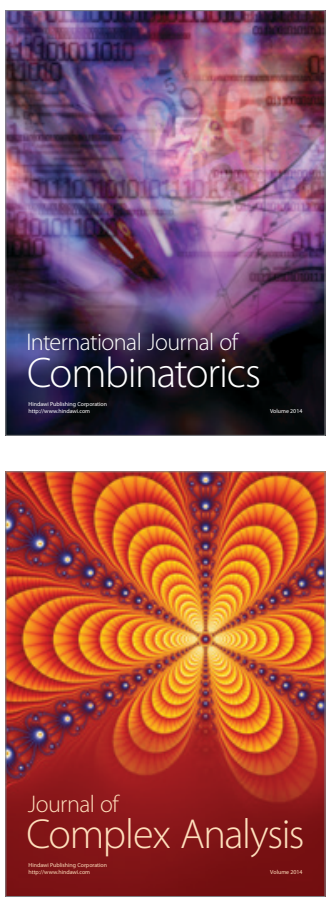

International Journal of

Mathematics and

Mathematical

Sciences
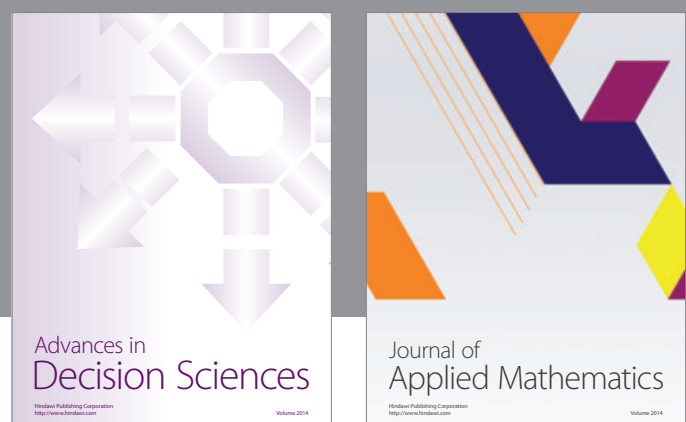

Journal of

Applied Mathematics
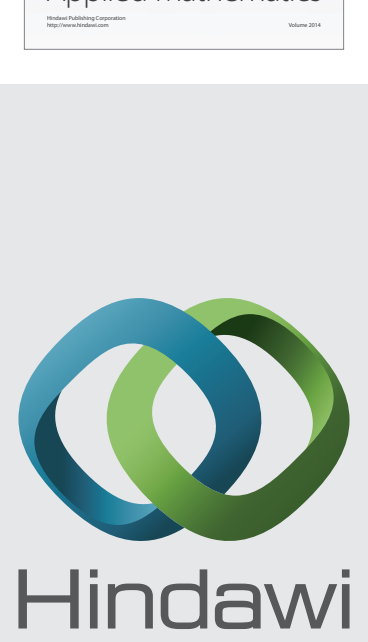

Submit your manuscripts at http://www.hindawi.com
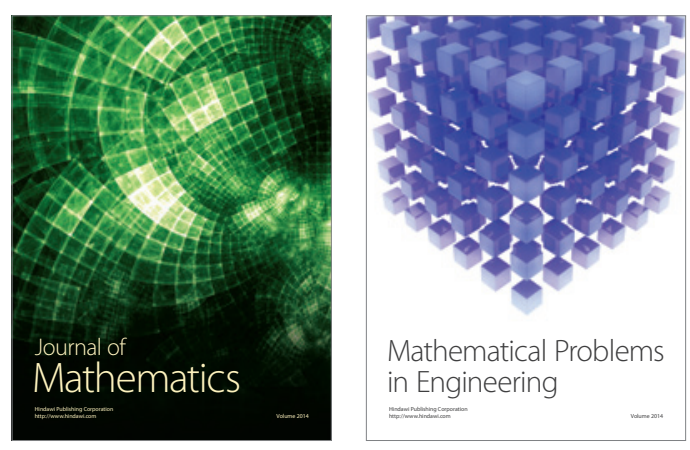

Mathematical Problems in Engineering
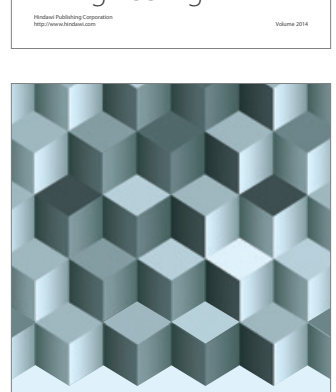

Journal of

Function Spaces
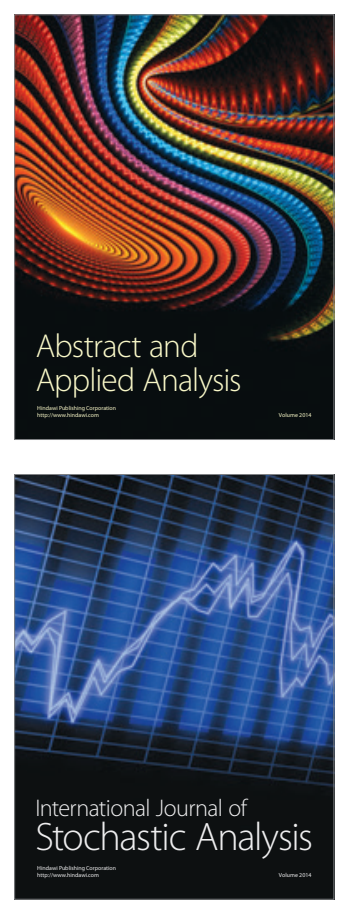

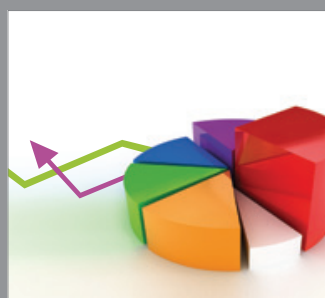

ournal of

Probability and Statistics

Promensencen
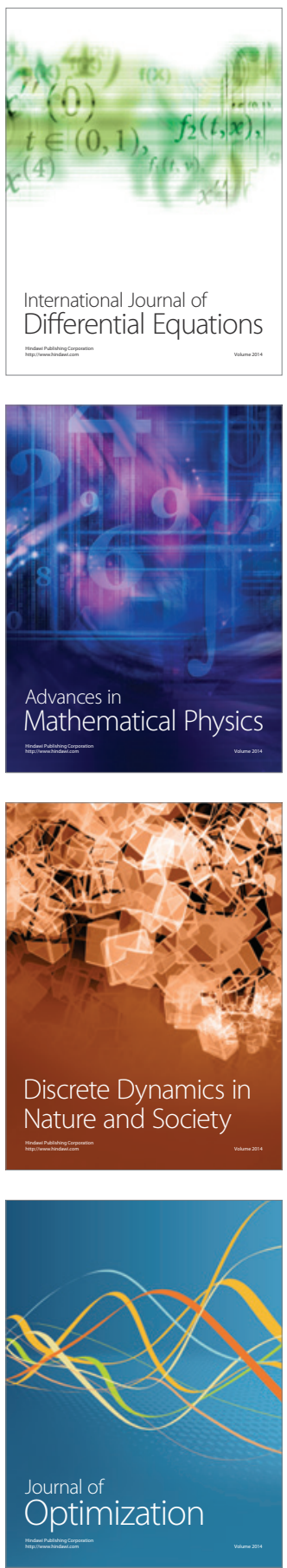